\title{
Activité anthelminthique du diacétate de plomb dibutyle
}

\author{
por G. GRAS
}

\begin{abstract}
RÉSUMÉ
Le diacétate de plomb dibutyle, composé organique du plomb, de structure $\mathrm{R}_{2} M X_{2}$, est doué, comme les composés de l'étain de structure similaire, d'une activité anthelminthique élevée.

L'activité de ce composé déterminée sur la souris expérimentalement parasitée par Hymenolepis fraterna est très supérieure en valeur absolve, à celle de la plupart des anthelminthiques connus à l'heure actuelle. Cette activité semble liée à l'affinité de ce dérivé pour les groupes - SH. La DL 50 per os chez la souris calculée par la méthode de BERHENS et KARBER, est de $85 \mathrm{mg} / \mathrm{kg}$.
\end{abstract}

Généralités
Les composés minéraux et organiques de l'étain et du plomb présentent de grandes analogies dans leurs propriétés physico-chimiques (16), (20). De même il a été démontré récemment que les propriétés biocides des dérivés trıalkylétain (16), (19) ainsi que certains aspects de leurs propriétés biochimiques (1), (2) se retrouvaient chez les dérivés trialkyl du plomb (3), (4). Certains composés organiques de l'étain ont une activité anthelminthique marquée sur les cestodes (KERR 1952, KERR et WALDE 1956, GRAS 1956). Cette activité est moximum pour les composés de structure $\mathrm{R}_{2} \mathrm{SnX}_{2}$ (schéma dans lequel $R$ est un radical aryl ou alkyl directement lié à l'atome d'étain et $X$ un reste anionique) et ceci lorsque $R$ et $X$ sont de structure simple (KERR ef WALDE 1956). Les meilleurs résultats ont été obtenus avec les dérıvés dibutyle (KERR 1952, CASTEL, HARANT et GRAS 1958, GRABER et GRAS 1962-1963).

La variation de $X$ n'entraîne que peu de changement dans l'activité, du moins si $X$ a un faible poids moléculaire; en définitive, comme le fait très justement remarquer $\operatorname{KERR}\left({ }^{*}\right)$, la

(*) KERR (K. B.). The toxicity of retrevalent tin compounds for poultry. Travail non publié, communiqué pendant le voyage de l'auteur aux U. S. A. en 1963. toxicité et l'activité des dérivés dibutylétain dépendent essentiellement du pourcentage de la molécule en dibutylétain.

Ces diverses considérations nous ont incité à rechercher l'activité anthelminthique éventuelle de dérivés dibutyle du plomb. Car du point de vue théorique, avant d'entreprendre une étude systématique des propriétés anthelminthiques des composés organo-plombiques, il nous paraissait extrêmement intéressant de savoir si le pouvoir cestocide maximum rencontré chez les dérivés dibutylétain apparaissait également chez le dibutylplomb.

\section{Matériel et Méthode}

\section{Produits utilisés :}

Le composé dibutylplomb choisi est le diacétate de plomb dibutyle, composé bien cristallisé de formule:<smiles>CCCC[Pb]([14CH3])(OC(C)=O)OC(C)=O</smiles>

P. F. $=54^{\circ} \mathrm{C}$. Le diacétate de plomb dibutyle est très soluble dans l'acétone, soluble dans de 
nombreux solvants organiques, Nous avons trouvé que la solubilité de ce produit dans l'eau à $20^{\circ} \mathrm{C}$. est de 5,96 p. 100 . Son poids moléculaıre est de P. M. $=439,32$.

Nous avons comparé l'activité du diacétate de plomb dibutyle à celle du maléate d'étain dibutyle car il ne nous a pas été possible pour le moment de nous procurer du diacétate d'étain dibutyle.

Nous avons choisi le maléate à cause de son poids moléculaire et de son pourcentage en étain très voisins de ceux du diacétate :

maléate d'étain dibutyle<smiles>CCCCC[Sn]1(CCCCC)OC(=O)C(=O)O1</smiles>

diacétate d'étain dibutyle

P. M. 346,82. \% en étain 34,22<smiles>CCCCC[Sn](CCCC)(CCCC)OC(C)=O</smiles>

P.M. $350,82 . \%$ en étain 33,84 .

Nous avons également déterminé dans le mêmes conditions l'activité de l'arsénıate d'étain (**) $\mathrm{SnHAsO}_{4}, 1 / 2 \mathrm{H}_{2} \mathrm{O}$, dont les propriétés anthelminthiques en médecine vétérinaire se sont avérées excellentes (GRAS et GRABER 1965).

\section{$2^{\circ}$ Détermination de l'activité anthelminthique :}

L'actıvité anthelminthique a été déterminée en utilisant la méthode de CAVIER (7). Une étude systématique menée au laboratoire de pharmacle chimıque ( $\mathrm{Pr}$ P. CASTEL), a montré que cette méthode est la mieux adaptée pour effectuer des «screening » sur des composés nouveaux (18). La technique est la suivante:

(*) Echantillon fourni par la B. X. Plastics L. D. T. Branthan Works Manigree Essex Angleterre.

(**) Préparé au laboratoire.
Des souris de souche R. A. P. agées de quatre semaines et exemptes de parasites sont expérimentalement infestées avec 50 œufs mûrs d'Hymenolepis fraterna. Pour cela, on prélève dans l'intestin de souris déjà parasitées, le dernier proglotis du strobile. Les proglotis sont ensuite dilacérés sur une lame de microscope, et mis en suspension dans du soluté physiologique à 9 p. 1000.

Les œufs sont alors numérés à la cellule de Nageotte ; puis on effectue une dilution convenable de manière à avolr 50 œufs mûrs dans $0,2.5 \mathrm{ml}$. Ce volume de solution est alors injecté per os aux souris à l'aide d'une canule veineuse montée sur une seringue précise. Quinze jours après on recherche les cufs de parasites dans les fèces des animaux d'expérience. Dans ces conditions on obtient, suivant les lots, un pourcentage de parasitation de 80 à 100 p. 100. Le nombre de vers varie également dans des proportions acceptables. Dans nos essais qui ont été faits sur 4 lots différents de souris, nous avons chaque fois gardé 20 souris témoins par lot. Chez ces 4 lots témoins le nombre moyen de vers a été au minimum de 5,4 et au maximum de 9,7 .

\section{$3^{\circ}$ Administration des produits :}

Le diacétate de plomb dibutyle est suffisament soluble dans l'eau pour être administré en solution aqueuse. Les diverses doses de ce produit sont injectées dans un volume de $0,25 \mathrm{ml}$.

Le maléate d'étain dibutyle a été injecté en suspension dans la gomme arabique et l'arséniate d'étaın en suspension dans l'Emulsov extra PSovilo. Ces deux composés sont également administrés sous le volume de $0,25 \mathrm{ml}$.

\section{Appréciation de l'activité :}

L'activité est appréciée par le nombre d'animaux complètement déparasités en fonction de la dose administrée.

\section{Résultats}

Des essais précédents nous ont montré que pour l'arséniate d'étain et le dichlorure d'éfain dibutyle (5), (11), la dose de $100 \mathrm{mg} / \mathrm{kg}$ était, suivant le degré d'infestation des animaux, voisine de la dose déparasitant complètement les animaux d'expérience. Nous avons done choisi 
cette dose comme point de départ de notre expérimentation. Les résultats montrent (voir tableau) que pour l'arséniate d'étain la dose de $100 \mathrm{mg} / \mathrm{kg}$ est suffisante pour obtenir une déparasitation complète des souris ce qui est normal, le nombre de vers étant moins élevé que dans nos essais précédents (GRAS et GRABER 1965).

Le maléate d'étain dibutyle permet d'obtenir 100 p. 100 pour une dose de $75 \mathrm{mg} / \mathrm{kg}$, mais on obtient encore un coefficient de 90 p. 100 pour une dose de $50 \mathrm{mg} / \mathrm{kg}$.

Quant au diacétate de plomb dibutyle, il montre une activité anthelminthique très élevée puisqu'on obtient une déparasitation complète pour une dose qussi faible que $5 \mathrm{mg} / \mathrm{kg}$. Ce composé est donc 15 fois plus actif que le maléate d'étain dibutyle et 20 fois plus actif que l'arsénıate d'étain.

L'augmentation de l'activité du dérivé dibutylplomb par rapport au dérivé dibutylétain est certainement liée au mécanisme d'action biochimique de ces composés. II a en effet été montré que les composés dialkylétain (STONER, BARNES et DUFF 1955, ALDRIGE 1955) et dialkylplomb (CREMER 1959-1961), agissent comme thioloprive. Les cestodes sont certainement très sensibles à ce type d'agent biochimique.

L'arséniate d'étain est un anthelminthique puissant pour la plupart des cestodes (GRAS et
GRABER 1965). Or nous avons montré que l'activité de l'arséniate d'étain étaił due presque en totalité à l'arsenic qu'il contient qui est le type même des composés thioloprives. Ce point de vue semble bien confirmé par les faits suivants :

- Tout d'abord, l'accroissement de l'activité pour le dibutylplomb n'est pas intrinsèquement dû au cation $\mathrm{Pb}$, car, déterminée dans les mêmes conditions sur $H$. froterna de la souris, l'activité anthelminthique de composés minéraux du plomb comme lacétate et l'oxyde ( $\mathrm{PbO}$ ) est nuile pour des doses de 100 à $500 \mathrm{mg} / \mathrm{kg}$. - Les dérivés $R_{3} M X$ qui sont sans effet sur les groupes - SH (CREMER 1959) devraient se montrer moins actifs comme cestocides bien que ce soit dans ce groupe que l'on rencontre les composés biocides les plus puissants $(12,13$, 16,19 ). Nous avons pu vérifier ceci en administrant à 10 souris une dose de $5 \mathrm{mg} / \mathrm{kg}$ d'acétate de plomb tributyle. A cette dose, qui pour le dérivé dibutyle permet d'obtenir un pourcentage de déparasitation à 100 p. 100, le dérivé tributyle est complètement inactif.

- Lorsqu'on donne à des souris une dose de $5 \mathrm{mg} / \mathrm{kg}$ de B. A. L. par voie orole trente minutes avant l'administration de $5 \mathrm{mg} / \mathrm{kg}$ de diacétate de plomb dibutyle, l'activité anthelminthique de ce produit devient nulle.

Activité anthelminthique du diacétate de plomb dibutyle, du maléate d'étain dibutyle et de 1'areániate d'étain sur Hymenoleníf fraterna

\begin{tabular}{|c|c|c|c|c|c|c|}
\hline Produits & $\begin{array}{l}\text { Doses } \\
\text { mg/kg }\end{array}$ & $\begin{array}{l}\text { N } \\
\text { de dé }\end{array}$ & fin & $\begin{array}{l}\text { Nombre } \\
\text { moyen } \\
\text { de, vers }\end{array}$ & $\begin{array}{l}\text { liombre de souris } \\
\text { completement } \\
\text { déparasitées }\end{array}$ & $\begin{array}{l}\text { Pourcentage } \\
\text { de } \\
\text { déparasitage }\end{array}$ \\
\hline Témoins & - & 80 & 78 & 8,1 & - & - \\
\hline $\begin{array}{l}\text { Maléate } \\
\text { d'étain } \\
\text { dibutyle }\end{array}$ & $\begin{array}{r}100 \\
75 \\
50 \\
25\end{array}$ & $\begin{array}{l}10 \\
10 \\
10 \\
10\end{array}$ & $\begin{array}{r}7 \\
8 \\
10 \\
10\end{array}$ & $\begin{array}{l}0 \\
0 \\
0,1 \\
2,7\end{array}$ & $\begin{array}{l}7 \\
8 \\
9 \\
4\end{array}$ & $\begin{array}{r}100 \\
100 \\
90 \\
40\end{array}$ \\
\hline $\begin{array}{l}\text { Diecétate } \\
\text { de plomb } \\
\text { dlbutyle }\end{array}$ & $\begin{array}{c}100 \\
75 \\
50 \\
25 \\
10 \\
5 \\
3 \\
1,5\end{array}$ & $\begin{array}{l}10 \\
10 \\
10 \\
10 \\
10 \\
10 \\
10 \\
10\end{array}$ & $\begin{array}{r}5 \\
8 \\
10 \\
10 \\
10 \\
10 \\
10 \\
10\end{array}$ & $\begin{array}{l}0 \\
0 \\
0 \\
0 \\
0 \\
0 \\
4,4 \\
5,5\end{array}$ & $\begin{array}{r}5 \\
8 \\
10 \\
10 \\
10 \\
10 \\
2 \\
0\end{array}$ & $\begin{array}{r}100 \\
100 \\
100 \\
100 \\
100 \\
100 \\
20 \\
0\end{array}$ \\
\hline $\begin{array}{l}\text { Arsénilate } \\
\text { d'étaln }\end{array}$ & $\begin{array}{r}100 \\
75 \\
50\end{array}$ & $\begin{array}{l}10 \\
10 \\
10\end{array}$ & $\begin{array}{l}10 \\
10 \\
10\end{array}$ & $\begin{array}{l}0 \\
1,6 \\
4,6\end{array}$ & $\begin{array}{r}10 \\
7 \\
1\end{array}$ & $\begin{array}{r}100 \\
70 \\
10\end{array}$ \\
\hline
\end{tabular}


Dans ces conditions il est possible que l'accroissement de l'activité cestocide du dibutylplomb par rapport au dibutylétain soit dô à la polarisation de la liaison $M-X$ qui augmente de l'éfain au plomb, ce qui peut se traduire par un accroissement de l'affinité de ce composé pour les liaisons $\mathrm{S}-\mathrm{H}$.

Cette question, ainsi que l'examen de l'activité anthelmınthique en fonction de la structure des composés organıques du plomb, fera l'objet d'une prochaine publication.

En ce qui concerne la toxicité, nous avons effectué une première estimation sur 6 lots de 20 souris (q) R. A. P. d'un poids de $20 \pm 2 \mathrm{~g}$. La DL 50 calculée par la méthode de BERHENS et KARBER à l'échéance de 8 jours est de $85 \mathrm{mg} /$ $\mathrm{kg} ;$ ce qui donne un coefficient chimiothérapique (Rapport de la D. E. 100 à la DL 50) de 17. Ce résultat est plus favorable que ceux obtenus avec l'arséniate d'étain (11) ef les composés organiques de l'étaın (5), (6), (10).

\section{Conclusion}

Les composés dibutyle de l'étain ef du plomb de structure $R_{2} M X_{2}(M=S n$ ou $P b)$, sont doués d'une activité anthelminthique élevée. Le diacétate de plomb dibutyle est beaucoup plus actif que les composés organiques de l'étain et que l'arséniate d'étain. A notre connaissance, i n'existe pas à l'heure actuelle, d'anthelminthique qui agisse à une dose aussi faible sur Hymenolepis fraterna de la souris.

Chez la souris le coefficient chimiothérapique est également supérieur à ceux obtenus avec l'arséniate d'étain ef les composés organiques de l'étain.
Ceci peut permettre d'espérer que des résultats favorables seront également enregistrés sur d'autres espèces animales. Les premiers résultats que nous venons d'obtenir sur le poulet expérimentalement parasité par Roilhetino ceshicllus sont très favorables puisque, à la dose de $5 \mathrm{mg} /$ $\mathrm{kg}$, le diacétate de plomb dibutyle permet de déparasiter complétement cet animal.

Bien entendu, l'application pratique du diacétate de plomb dibutyle en médecine vétérinaire dépendra des coefficients chimiothérapiques obtenus sur les espèces visées.

Enfin il ne laut pas perdre de vue, que l'utilisation des composés du plomb, même en médecine vétérinaire, risque de poser de sérieux. problèmes toxicologiques.

Cependant, I'utilisation sans inconvénient, depuis plusieurs années de l'arséniate de plomb (11) monntre que ce genre de problème peut avoir une solution.

\section{REMERCIEMENTS}

Nous adressons nos remerciements au Dr SCHRADE F. RADTKE Directeur de l'international Lead Zinc Research organisation New-York qui nous a adressé les échantillons des composés organiques du plomb utilisés dans notre expérimentation.

Nous remercions également le Dr Van Der WANT de l'Institut de chimie organique T. N. O. d'Utrecht qui nous a transmis ces échantillons ainsi que de nombreux renseignements les concernant.

\section{Faculté de Phormacie de Montpellier Laboratoire de Pharmacie Chimique P. Castel. Professeur}

\section{SUMMARY}

\section{Antheiminthic activity of dibutyllead diacetate}

Dibutyllead diacetate, a $R_{2} M X_{2}$ structure lead organic compound, is endowed with a high anthelminthic activity, as similar structure tin compounds. This compound activity determined in mice experimentally infested with Hymenolepis froterna is, in absolute value, above the activity of most of now known anthelminthics. This activity seems in connection with this derivative affinity towards - SH groups. LD 50, calculated by KARBER and BERHENS method, is $85 \mathrm{mg} . / \mathrm{kg}$. 


\section{RESUMEN}

Actividad antihelmintica del diacetato de plomo dibutilo

El diacetato de plomo dibutilo, compuesto orgánico del plomo, de estructura $R_{2} M X_{2}$, tiene, asi como los compuestos del estaño de estructura similar, una actividad antihelmíntica importante. La actividad de este compuesto determinada en el ratón, parasitado experimentalmente por Hymenolepis fraterna, es muy superiora en cuanto a la valor absoluta a la de la mayor parte de los antihelmínticos conocidos acłualmente. Esta acividad parece ligada con la afinidad de este derivado para los grupos - SH. La DL 50 calculada mediante el método de BERHENS y KARBER, es de $85 \mathrm{mg} . \mathrm{kg}$.

\section{BIBLIOGRAPHIE}

(1) ALDRIDGE (W. N.) and CREMER (J. E.). The biochemistry of organotin compounds. Diethyltin dichloride and trietyltinsulphate. Blochem. J. 1955, 6I, (3) : 406-18.

(2) BARNES (J. $\dot{M}$.) and STONER (H. B.). The toxicology of tin compounds. Pharmacol. Rev. 1959, II : 211-31.

(3) CREMER (J. E.). - Biochemical studies on the toxicity of tetraetyllead and other organo-lead compounds. Brit. J. Industr. med. 1959, $16: 191-9$.

(4) CREMER (J. E.). - The toxicity of tetraetyl lead and related alkyl metallic compounds. Ann. Occup. Hyg. 1961, 3 : 226-30.

(5) CASTEL (P.), HARANT (H.) et GRAS (G.). Les possibilités anthelminthiques des composés organiques de l'étain. Thérapie, 1958, $13: 865-72$.

(6) CASTEL (P.), GRAS (G.) et BEAULATON (S.). - Recherches sur l'activité anthelminthique ef la toxicité de l'oxyde d'étain. Diphényle. Rev. Path. génér. et de Physiol. Clin. 1960, (715) : 235-43.

(7) CAVIER (R.). - - Sur une nouvelle technique pharmacologique d'essais des tenifuges. Ann. Pharm. fr. 1956, 14:595-552.

(8) GRABER (M.) et GRAS (G.). - Etude de l'activité anthelminthique et de la toxicité de quelques composés organiques de l'étain. I. - Dilaurate d'étain dibutyle. Rev. Elev. Méd. vét. Pays trop. $1962,15(4): 411-26$.

(9) GRABER (M.) et GRAS (G.). - Etude de l'activité anthelminthique et de la toxi- cité de quelques composés organiques de l'étain. II. - Maléate d'étain dibutyle. Rev. Elev. Med. Vét, Pays trop. 1963, $16(4): 427-38$.

(10) GRAS (G.). - L'étain. Etude expérimentale du pouvoir anthel minthique de quelques composés minéraux et organiques. Thèse Pharmacie Montpellier 1956.

(11) GRAS (G.) et GRABER (M.). - Les arséniates métalliques en médecine vétérinaire ; l'arséniate d'étain en particulier. - Comparaison avec d'autres tenifuges modernes. Rev. Elev. Med. Vet. Pays trop. 1964, 17 (4) 663-719.

(12) KAARS SIJPESTEIJN (A.), RIJKENS (F.) LUIJTEN (J. G. A.) et WILLEMSENS (K.). - Anatomie van Leeuwenhoeck. (J. microbiol. and Serol.) 1962, $28: 346-56$.

(13) KAARS SIJPESTEIJN (A.), RIJKENS (F.), VAN DER KERK (G. S. M.) and MANTEN (A.). - Nature, Lond. 1964, 201 : 736.

(14) KERR (K. B.). - Butynorate an effective and safe substance for the removal of Raillietina cesticillus from Chickens. Poultry. Sci. 1952, 31 : 328-336.

(15) KERR (K. B.) and WALDE (A. W.), - The anthelmintic activity of tetravalent tin compounds. Exp. Parasitol. 1956, 5(6): 560-70.

(16) LUIJTEN (J. G. A.) and VAN DER KERK (G. J. M.). - Investigation in the field of organotin chemistry. Tin Research Institute 1955 : 133 pages, 204 réf.

(17) STONER (H. B.), BARNES (J.M.) and DUFF (J. I.). - Studies on the toxicity of alkyl tin compounds. Brit. J. Pharmacol. 1955, $10: 16-22$. 
(18) UN SAROEUNG. — Thèse Pharmacie en préparation.

(19) VAN DER KERK (G. J. M.). - Quelques' recherches dans le domaine des composés organiques de l'étain. Chim. et Industr. 1963, 90 (3) : 251-8.
(20) WILLEMSENS (L. C.). - Organolead chemistry 111 pages, 421 réf. Insitute for organic chemestry T.N.O. Utrecht; published on behalf of the International Zinc Lead Research organization. Edité par Semper Avanti N. V. The Hague 1964. 